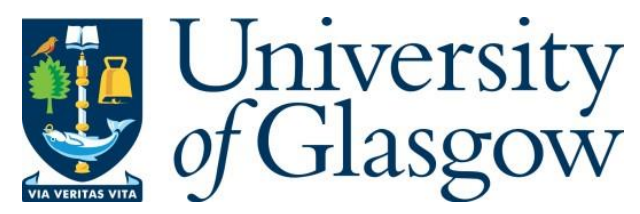

Onireti, O., Imran, M. A., and Heliot, F. (2011) On Achievable Rate Region of Multiple Coordinated Multiple Access Channels. In: 2011 IEEE 73rd Vehicular Technology

Conference (VTC Spring), Budapest, Hungary, 15-18 May 2011, ISBN 9781424483310.

There may be differences between this version and the published version. You are advised to consult the publisher's version if you wish to cite from it.

http://eprints.gla.ac.uk/137111/

Deposited on: 16 February 2017

Enlighten - Research publications by members of the University of Glasgow http://eprints.gla.ac.uk 


\title{
On Achievable Rate Region of Multiple Coordinated Multiple Access Channels
}

\author{
Oluwakayode Onireti, Muhammad Ali Imran and Fabién Heliot \\ Centre for Communications Systems Research, Guildford, University of Surrey \\ Guildford, United Kingdom \\ Email: \{o.onireti, m.imran, f.heliot\}@ surrey.ac.uk
}

\begin{abstract}
Coordination between two or more multiple access channel (MAC) receivers can enlarge the achievable rate region of the whole system. This paper focuses on coordination by sharing the codebooks of the users between the receivers of MACs. We first define the achievable rate region of the time invariant multiple coordinated MAC (MCMAC) and subsequently derive its achievable rate region. We later express the achievable rate region in terms of the dominating points. We base our numerical analysis on the two-user two-receiver Gaussian coordinated MAC and make comparison with the interference channel, full cooperation and the individual MAC performance analysis. It is observed that this approach though suboptimal is less complex in comparison with full cooperation and that the MCMAC rate region is at least equal to the rate region of the uncoordinated approach. Over several channel states, the rate region of MCMAC exceeds that of the uncoordinated approach.
\end{abstract}

Keywords- Coordination, cooperation, multiple access channel, achievable rate region.

\section{INTRODUCTION}

The capacity region for multiple access channel (MAC) is well established for various types of services, channel models, CSIT availability and nodes antenna configurations (single antenna, multiple antennas) such as; time invariant capacity, ergodic capacity, outage capacity, delay limited capacity, minimum rate capacity [1]-[7] and references therein. The MAC represents only a theoretical model for the uplink of a single cell wireless cellular system. The typical wireless cellular system is made up of several cells which for an uplink scenario lead to several MACs interfering with each other. Thus, a typical wireless communication system has similarities with an interference channel whose capacity region is known only for cases with strong interference [8-10] and very strong interference [11]. For other cases, an estimate of the capacity region to within a constant number of bits is known $[12,13]$.

Information theory has revealed that joint decoding of all signals at a central processor is the optimal approach for dealing with interference in cellular networks [14-17]. We refer this approach as joint processing MAC (JPMAC). JPMAC requires a high capacity backhaul to gather all the received signals at the central processor. It also incurs delay in users' signal decoding due to the signal gathering.

Multiple coordinated MAC (MCMAC) is introduced in this paper as an approach for reducing JPMAC limitations. By coordination we mean that all the MAC receivers can exchange some information about users including codebooks and their decoding outcome. This exhange can be a full or a partial exchange of relevant information. The exhanged information can be classified into two categories; system status which defines the state of the system and decision status which defines the encoding and decoding policies. Exchanged system status information could include users channel state information and their codebooks. Decision status include decoding order in the cell, transmit power of users, and decoding outcome of the receivers.

In MCMAC, we assume that each receiving node performs a local decoding of a subset of users but the receivers are allowed to share a properly chosen subset of control and state variables. Such an approach in contrast to JPMAC, which is equivalent to a single MAC, will lead to multiple MACs (each associated with a receiving node) that can perform some level of coordination through the exchange of necessary control and state variables. In a typical cellular radio communication terminology, sharing of only control and state variables implies a common control plane between cells' access points while the data planes are kept separate. Limiting shared information only to control plane will considerably reduce the backhaul load. It will also allow for fast decoding of users as each receiver has its own local decoding. Moreover, in contrast to JPMAC, the system does not need to wait to gather all the received signals at one central node or to exchange decoded data, e.g. through a conferencing approach.

The remaining part of this paper is organized as follows: In section II, we present the system model for the MCMAC. Then in Section III, we introduce theorems and utilize them for deriving the achievable rate region of the MCMAC. Section IV presents some simulations results which show some performance improvement when using MCMAC over interference channel and single MAC. Finally, Section V summaries our work.

\section{SYSTEM MODEL}

We consider $N$ coordinated MACs consisting of $N$ receiving nodes and $K$ users. We denote the set of receiving nodes by $\mathrm{N}=\{1,2, \ldots, N\}$ and the set of users by $\mathrm{K}=\{1,2, \ldots, K\}$. Every receiving node is assumed to know all the users' codebooks and will attempt to decode as many users as possible. The 


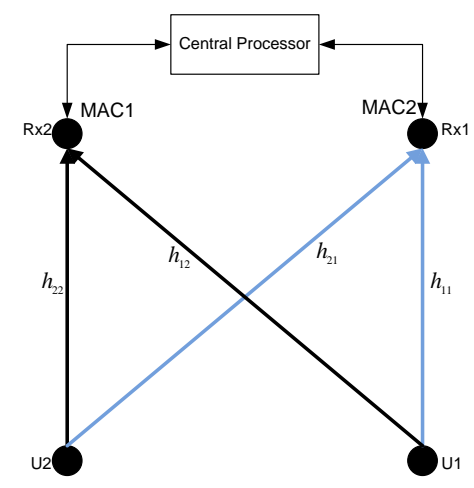

Fig. 1:Schematic diagram of a two-user coordinated MAC

receivers are allowed to share some control information mainly related to users' CSI through backhaul. Also a central processor (CP) is assumed to decide about users' transmission rate and the transmission format, i.e. users' codebooks. The decision outcome is then made available to all the receivers.

Definition 2.1: We define MCMAC by:

○ Multi-point to multi-point channel ( $\prod_{k \in \mathrm{K}} \Xi_{k}, p(\boldsymbol{y} \mid \boldsymbol{x})$, $\left.\prod_{n \in \mathrm{N}} \Psi_{n}\right)$ with $\boldsymbol{x} \in \prod_{k \in \mathrm{K}} \Xi_{k}$ and $\boldsymbol{y} \in \prod_{n \in \mathrm{N}} \Psi_{n}$ as input and output of the channel, respectively. In addition, $\Xi_{k}$ is the alphabet of input node $k$ and $\Psi_{n}$ is the alphabet of the output node $n$.

○ User message sets $\Omega_{k}=\left\{1,2, \ldots, 2^{v R}{ }^{k}\right\}$ for every $k \in \mathrm{K}$

$\circ$ User encoders $f_{k}^{(v)}: \Omega_{k} \rightarrow \Xi_{k}^{(v)}$ for every $k \in \mathrm{K}$

○ Decoders $g_{n}{ }^{(v)}: \Psi_{n}{ }^{(v)} \rightarrow \Omega_{k}$ for every $n \in \mathrm{N}$ and every $k \in \mathrm{K}_{n}$, where $\mathrm{K}_{n}$ is the set of users decoded by the receiver $n$,

where $v$ is the code length.

Code $\left(f_{k}{ }^{(v)}, g_{n}{ }^{(v)}, v\right)$ uses the channel for $v$ subsequent times. User $k$, generates the codeword $x_{k}\left(w_{k}\right)=f_{k}^{(v)}\left(w_{k}\right)$ by using encoder $f_{k}^{(v)}$ and its message $w_{k}$ and transmits it over the channel. The receiver $n$ upon reception of the sequence $y_{n} \in \Psi_{n}{ }^{v}$ carries out its decoding: $\hat{w}_{k}{ }^{(n)}=g_{n}{ }^{(v)}\left(y_{n}\right)$ for every $k \in \mathrm{K}_{n}$. Now if we denote $\mathrm{N}_{k}$ as the set of receivers attempting to decode user $k$, i.e. $\mathrm{N}_{k} \cong\left\{n: k \in \mathrm{K}_{n}\right\}$ then the user $k$ is said to be decoded erroneously if one of the receivers $n \in \mathrm{N}_{k}$ has failed to decode it correctly, i.e. there exist an $n \in \mathrm{N}_{k}$ with $\hat{w}_{k}{ }^{(n)} \neq w_{k}$. Then, user $k$ probability of error is defined as $P_{e, k}=\operatorname{Pr}\left\{\hat{w}_{k}^{(n)} \neq w_{k}\right.$ for some $\left.n \in \mathrm{N}_{k}\right\}$.

Definition 2.2: The rate set $\boldsymbol{R}=\left(R_{1}, \ldots, R_{K}\right)$ is said to be achievable by MCMAC if there is a sequence of codes $\left(f_{k}^{(v)}\right.$, $\left.g_{n}{ }^{(v)}, v\right)$ such that $\cup_{n \in \mathrm{N}} \mathrm{K}_{n}=\mathrm{K}$ and for every $k \in \mathrm{K}, P_{e, k}$ approaches 0 for large enough value of $v$.

\section{ACHIEVABLE RATE REGION OF CONSTANT MULTIPLE COORDINATED MULTIPLE ACCESS CHANNEL}

We here derive the achievable rate region of the time invariant MCMAC.
Definition 3.2: $C_{\mathrm{U}}^{(n)}$ is the rate region for receiver $n$ where every $k \in \mathrm{Y}$ with $\mathrm{Y} \subseteq \mathrm{K}$ is decodable at receiver $n$ and the rest of the users $k \in \mathrm{Y}^{c}$ are not attempted to be decoded at receiver $n$, i.e. act as extra noise at receiver $n$ :

$$
\mathrm{X}_{\mathrm{Y}}^{(n)}:=\left\{\boldsymbol{R}: \boldsymbol{R}(\Sigma) \leq \mathrm{I}\left(\boldsymbol{X}_{\Sigma} ; \boldsymbol{Y} \mid \boldsymbol{X}_{\Sigma^{c} \cap \mathrm{Y}}\right) \text { for all } \Sigma \subseteq \mathrm{Y}\right\},
$$

for a given input distribution $\prod_{k \in \mathrm{K}} p_{k}\left(x_{k}\right)$, where $\boldsymbol{R}(\Sigma)=\sum_{k \in \Sigma} R_{k}$ and $\boldsymbol{X}_{\Sigma}=\left\{X_{k}: k \in \Sigma\right\} . \mathrm{C}_{\mathrm{U}}^{(n)}$ is the conventional well established MAC capacity region with user sets $\mathrm{Y}$ and, therefore, it is correct to state that users in the set $\mathrm{Y}$ are decodable by receiver $n$. The user signals that are not decoded at receiver $n$ must be decodable in at least one of the remaining receivers.

Lemma 3.3: if $\Sigma_{1} \subseteq \mathrm{K}$ and $\Sigma_{2} \subseteq \mathrm{K}$ are both decodable by a receiver $n$ for a given rate vector $\boldsymbol{R}$, then $\Sigma_{1} \cup \Sigma_{2}$ is also decodable by that receiver $n$.

Proof: The receiver can employ two parallel decoders one for decoding the users in $\Sigma_{1}$ and the other one for decoding the users in $\Sigma_{2}$; therefore, all the users in $\Sigma_{1} \cup \Sigma_{2}$ will be decoded in the end. Lemma 3.3 leads us to the definition of the maximal decodable set:

Lemma 3.4: if $\boldsymbol{R} \int \mathrm{X}_{\Sigma_{1}}^{(n)}$ and $\boldsymbol{R} \int \mathrm{X}_{\Sigma_{2}}^{(n)}$ then $\boldsymbol{R} \int \mathrm{X}_{\Sigma_{1} \cup \Sigma_{2}}^{(n)}$

Proof: Let us assume a given decoding order at the receiver such that $\boldsymbol{R} \int \mathrm{X}_{\Sigma_{1}}^{(n)}$ and $\boldsymbol{R} \int \mathrm{X}_{\Sigma_{2}}^{(n)}$ are achievable. From lemma 3.3, if $\mathrm{X}_{\Sigma_{1}}^{(n)}$ and $\mathrm{X}_{\Sigma_{2}}^{(n)}$ are achievable then $\mathrm{X}_{\Sigma_{1} \cup \Sigma_{2}}^{(n)}$ is achievable. There exists some decoding order in $X_{\Sigma_{1} \cup \Sigma_{2}}^{(n)}$ that satisfies the achievability of $\boldsymbol{R}$

Lemma 3.5: $\Sigma_{1} \subset \Sigma_{2}$ does not imply $\mathrm{X}_{\Sigma_{1}}^{(n)} \subset \mathrm{X}_{\Sigma_{2}}^{(n)}$.

Proof: Given that $\|$ denotes the cardinality of a set. The rate region $X_{\Sigma_{1}}^{(n)}$ is bounded in the dimension of $\left|\Sigma_{1}\right|$ and unbounded in $\left|\Sigma_{2}\right|-\left|\Sigma_{1}\right|$ while the rate region of $X_{\Sigma_{2}}^{(n)}$ is bounded in the dimension $\left|\Sigma_{2}\right|$. Thus we cannot say that $\mathrm{X}_{\Sigma_{1}}^{(n)} \subset \mathrm{X}_{\Sigma_{2}}^{(n)}$.

Definition 3.6: For a given rate vector $\boldsymbol{R}$, the maximal decodable set $\mathrm{K}_{n}(\boldsymbol{R})$ by a receiver $n$ is the set that contains all the decodable sets of the receiver $n$ such that if $\mathrm{K}_{n}^{\prime}$ is decodable by the receiver $n$ then $\mathrm{K}_{n}^{\prime} \subseteq \mathrm{K}_{n}(\boldsymbol{R})$.

The definition implies that the maximal decodable set at a receiver is a superset whose subsets are also decodable at the same receiver.

Corollary 3.7: Rate $\boldsymbol{R}$ is achievable if its decodability satisfies: $\mathrm{UK}_{n}(\boldsymbol{R})=\mathrm{K}$

Proof: Corollary 3.7 satisfies definition 3.1 which requires that a user must be decodable in at least one of the receivers such that each user $k$ must belong to at least one $\mathrm{K}_{n}$. Therefore, the union of the decodable sets over the entire receiver must 
correspond to the set of all the users K. Thus, corollary 3.7 is satisfied.

Based on the earlier definitions, lemmas and corollary we present a theorem that defines the achievable rate region of MCMAC

Theorem 3.8: The achievable rate region of constant MCMAC $\Theta$ is given as

$$
\begin{aligned}
Q & =\underset{\left\{\mathrm{K}_{1}, \mathrm{~K}_{2}, \ldots, \mathrm{K}_{N}\right\}}{\operatorname{Co}}\left\{\bigcap_{n \in \mathrm{N}} \mathrm{C}_{\mathrm{K}_{n}}^{(n)}\right\} \text { s.t. } \bigcup_{n \in \mathrm{N}} \mathrm{K}_{n}=\mathrm{K} \\
& =\left\{\boldsymbol{R}: \bigcup_{n \in \mathrm{N}} \mathrm{K}_{n}(\boldsymbol{R})=\mathrm{K}\right\},
\end{aligned}
$$

where $C o$ represents the convex hull operation. We present a proof of this theorem after defining the dominating points and the extreme points of the achievable rate region of MCMAC

Definition 3.9: A rate vector $\boldsymbol{R}$ will dominate another rate vector $\boldsymbol{R}^{\prime}$ iff $R_{k} \geq R_{k}^{\prime} \forall k \in \mathrm{K}$.

Figure 2 shows the dominating points a, $b, c, d$ and $e$ with point $f, g, h, l, m$ and $o$ being dominated.

For a given set of decodability sets $\left\{\mathrm{K}_{1}, \mathrm{~K}_{2}, \ldots, \mathrm{K}_{N}\right\}$ the achievable sub-region is given by $\bigcap_{n \in \mathrm{N}} \mathrm{C}_{\mathrm{K}_{n}}^{(n)}$. The projection of region $\mathrm{C}_{\mathrm{K}_{n}}^{(n)}$ over subspace spanned by users in $\mathrm{K}_{n}$ will be a polymatroid and thus will be defined by its $\left|\mathrm{K}_{n}\right|$ ! vertices. Consequently under the condition $\cup_{n \in \mathrm{N}} \mathrm{K}_{n}=\mathrm{K}$ the region $\bigcap_{n \in \mathrm{N}} \mathrm{C}_{\mathrm{K}_{n}}^{(n)}$ will be bounded and will become a polyhedron [4].

As a result this region will also be defined by a limited number of vertices. Every point within this region will be dominated by one of its vertices. Let us denote the set of all the vertices by $\mathrm{P}=\left\{\boldsymbol{R}_{1}^{*}, \boldsymbol{R}_{2}^{*}, \ldots\right\}$ where $\boldsymbol{R}_{\mathrm{i}}^{*}$ is the rate vector of the $\mathrm{i}^{\text {th }}$ vertex.

The achievable rate region of constant MCMAC can also be represented as the convex closure of all dominating point i.e.

$$
\Theta=\underset{\left\{\mathrm{K}_{1}, \mathrm{~K}_{2}, \ldots, \mathrm{K}_{\mathrm{N}}\right\}}{C o} \mathrm{P} \quad \text { s.t. } \bigcup_{n} \mathrm{~K}_{n}(\boldsymbol{R})=\mathrm{K}
$$

Definition 3.10: The extreme points of the 2-user 2-receiver MCMAC model illustrated in figure 1 can be obtained by decomposing the MCMAC into two MACs, which are MAC1 and MAC2. Based on the definition of extreme points of MAC in [4], two extreme points can be identified for each MAC. User2 for example has two extreme points, one in MAC2 $p 11$ with user2 achieving $R_{2}^{(2)}$ and another $p 12$ in MAC1 with

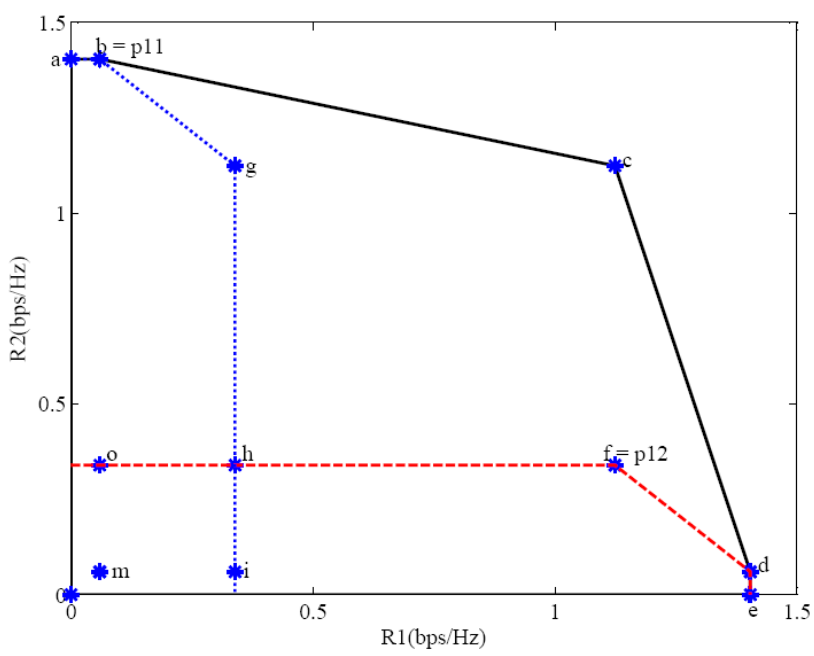

Fig. 2. Dominant points and extreme points on the achievable rate region of MCMAC

user2 achieving $R_{2}^{(1)}$. The extreme point of user $k$ in the MCMAC rate region is given as

$$
R_{k}^{(E x)}=\max _{\mathrm{N}}\left(R_{k}^{(n)}\right) .
$$

Thus for user2, $R_{2}^{(E x)}=\max \left(R_{2}^{(2)}, R_{2}^{(1)}\right)$ which is shown in figure 2 as point $p 11$.

Using the definitions above, we can now prove theorem 3.8

Proof of theorem 3.8: We prove the theorem for a simplified model with two receivers and then generalize for $K$ user and $N$ receivers.

In order to prove this theorem we consider two scenarios; in the first Scenario, case 1, we assume that the receivers can successfully decode all the messages of all the users K. In case 2 , the receivers can only decode a subset of all the users $\mathrm{K}$ due to path loss, fading or the receiver has only the codebook of a subset of users.

The capacity region of a discrete memoryless MAC with probability transition matrix $p\left(y \backslash x_{1}, \ldots, x_{m}\right)$ is given in [1] as the set of rate vectors $\boldsymbol{R}$ that satisfies

$$
\boldsymbol{R}(\Sigma) \leq I\left(\mathbf{X}_{\Sigma} ; \mathbf{Y} \mid \mathbf{X}_{\Sigma^{c} \cap \mathrm{K}}\right) \quad \forall \Sigma \subseteq \mathrm{K}
$$

Case 1: Let us Consider that $|\mathrm{K}|=2, \mathrm{~K}_{1}$ is the set of users decodable at receiver 1 and $\mathrm{K}_{2}$ is the set of users decodable at receiver 2. We index User 1 and User 2 as 1 and 2 respectively. To satisfy the definition of the achievable rate of MCMAC, the following combination $\left\{\mathrm{K}_{1}, \mathrm{~K}_{2}\right\}$ can be satisfied : $\{\{1\},\{2\}\},\{\{2\},\{1\}\},\{\{1\},\{1,2\}\},\{\{2\},\{1$, $2\}\},\{\{\},\{1,2\}\},\{\{1,2\},\{1\}\},\{\{1,2\},\{2\}\},\{\{1,2\},\{\}\}$, $\{\{1,2\},\{1,2\}\}$.

Observe that for each $\mathrm{K}_{n}$ we can obtain the rate region for $\{1\}$, $\{2\}$ and $\{1,2\}$ in this approach we treat the users not in the set $\mathrm{K}_{n}$ as noise. For $\mathrm{K}_{1}$ we represent the region with $\mathrm{T}_{1}, \mathrm{~T}_{2}, \mathrm{~T}_{1,2}$ and for $\mathrm{K}_{2}$ we represent the region with $\varsigma_{1}, \varsigma_{2}, \varsigma_{1,2}$, where $T_{1}$, $\mathrm{T}_{2}, \mathrm{~T}_{1,2}, \varsigma_{1}, \varsigma_{2}$ and $\varsigma_{1,2}$ satisfy

$$
\mathrm{T}_{1} \leq I\left(X_{1} ; Y_{1}\right)
$$




$$
\begin{aligned}
& \mathrm{T}_{2} \leq I\left(X_{2} ; Y_{1}\right) \\
& \mathrm{T}_{1,2}=\left\{\boldsymbol{R}: \mathrm{R}(\mathrm{S}) \leq I\left(X_{s} ; Y_{1}\right) \mid X_{s^{c} \cap \mathrm{K}_{n}}\right\} \forall S \subseteq \mathrm{K}_{n}=\{1,2\} \\
& \varsigma_{1} \leq I\left(X_{1} ; Y_{2}\right) \\
& \varsigma_{2} \leq I\left(X_{2} ; Y_{2}\right) \\
& \varsigma_{1,2}=\left\{\boldsymbol{R}: \mathrm{R}(\mathrm{S}) \leq I\left(X_{s} ; Y_{2}\right) \mid X_{s^{c} \cap \mathrm{K}_{n}}\right\} \forall S \subseteq \mathrm{K}_{n}=\{1,2\}
\end{aligned}
$$

The intersection of all possible combination of the rate $\mathrm{T}$ and $\varsigma$ that satisfies definition 2.2 are achievable in MCMAC rate region for the two-user two-receiver scenario. For example

$$
\begin{aligned}
& \varsigma_{1} \cap \mathrm{T}_{2}, \varsigma_{2} \cap \mathrm{T}_{1}, \mathrm{~T}_{1,2}, \varsigma_{1,2}, \varsigma_{1,2} \cap \mathrm{T}_{1,2}, \varsigma_{1} \cap \mathrm{T}_{1,2}, \\
& \varsigma_{2} \cap \mathrm{T}_{1,2}, \varsigma_{1,2} \cap \mathrm{T}_{1}, \varsigma_{1,2} \cap \mathrm{T}_{2}, \varsigma_{1,2} \text { and } \mathrm{T}_{1,2}
\end{aligned}
$$

are all achievable in the rate region

In order to obtain the boundary of the achievable rate region, the concept of dominating points in section 3.9 can be applied. The dominating point over all possible combination of $\mathrm{T}$ and $\varsigma$ that satisfies definition 2.2 is obtained. The boundary of the rate region can thus be obtained through performing time sharing between the dominating points which implies that the convex closure of $\mathrm{P}$ is achievable. QED

Case 2: Let us consider that $|\mathrm{K}|=2, \mathrm{~K}_{1}$ is the set of users decodable at receiver 1 and $\mathrm{K}_{2}$ is the set of users decodable at receiver 2 with the constraint that user 2 cannot be decoded at receiver 1 . This implies that user 2 always act as noise to receiver 1 . To satisfy the definition of the achievable rate of MCMAC, the following combination $\left\{\mathrm{K}_{1}, \mathrm{~K}_{2}\right\}$ can be satisfied:

$\{\{1\},\{1,2\}\},\{\{1\},\{2\}\},\{\{\},\{1,2\}\}$. Consequently, we can obtain the achievable rate of $\{1\}$ for each $\mathrm{K}_{1}$, whereas, we can obtain the rate region of $\{2\}$ and $\{1,2\}$ for $\mathrm{K}_{2}$. In this approach we treat the users not in the set $\mathrm{K}_{n}$ but in as noise. For $\mathrm{K}_{1}$ we represent the region with $\mathrm{T}_{1}$, and for $\mathrm{K}_{2}$ we represent the region with $\varsigma_{2}, \varsigma_{1,2}$. Where $T_{1}, \varsigma_{2}$ and $\varsigma_{1,2}$ satisfy

$$
\begin{gathered}
\mathrm{T}_{1} \leq I\left(X_{1} ; Y_{1}\right) \\
\varsigma_{2} \leq I\left(X_{2} ; Y_{2}\right) \\
\varsigma_{1,2}=\left\{\boldsymbol{R}: \mathrm{R}(\mathrm{S}) \leq I\left(X_{s} ; Y_{2}\right) \mid X_{s^{c} \cap \mathrm{K}_{n}}\right\} \forall S \subseteq \mathrm{K}_{n}=\{1,2\}
\end{gathered}
$$

The intersection between all the possible combination of the rates $\mathrm{T}$ and $\varsigma$ that satisfy definition 2.2 are achievable in MCMAC rate region for the three-user two-receiver scenario. For example $\varsigma_{2} \cap \mathrm{T}_{1}, \varsigma_{1,2} \cap \mathrm{T}_{1}$ and $\varsigma_{1,2}$ are all achievable. As in case 1, the dominating point and time sharing concepts are applied to obtain the boundary of the rate region.

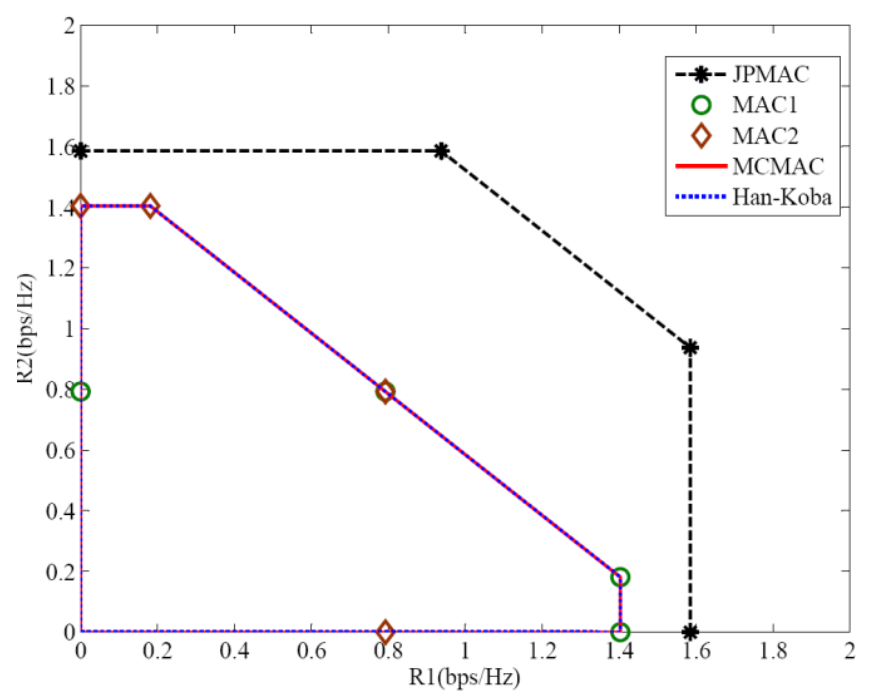

Fig. $3 P_{1}=P_{2}=6, h_{12}=h_{21}=1 / 3$

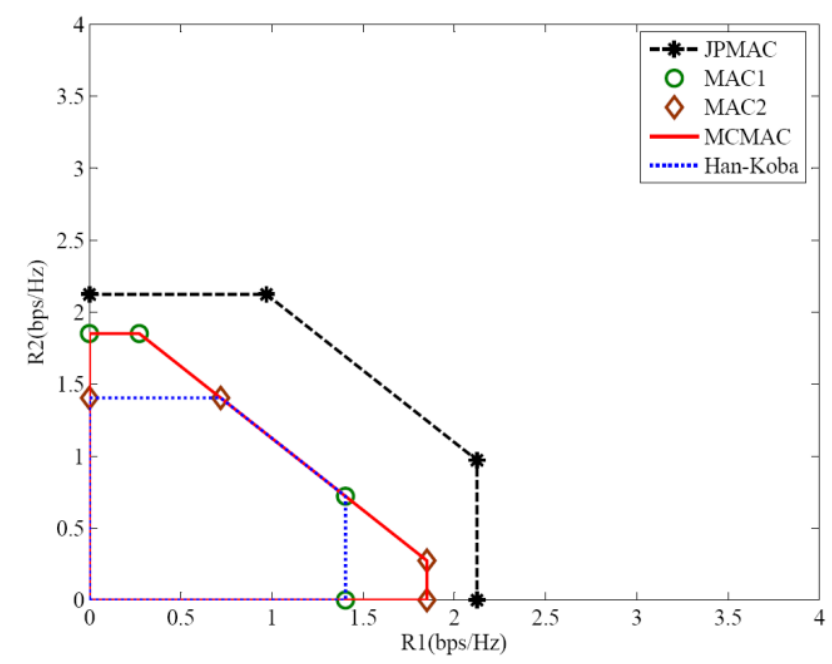

Fig. 4. $P_{1}=P_{2}=6, h_{12}=h_{21}=2$

\section{SimUlating THE ACHIEVABLE RATE REGION}

We consider the Gaussian MCMAC with $h_{11}$ and $h_{22}$ normalized to standard form as in $[10,11]$. The results for case 1 with two users and two receivers are illustrated in Figs. 3-5, where JPMAC, MAC1, MAC2, MCMAC and Han-Koba rate regions are compared. The JPMAC is the rate region that is obtained by joint processing of the signals when the backhaul have an unlimited delay-less capacity which is connected to a central processor. MAC1 and MAC2 are the rate regions of receivers 1 and 2. MCMAC achievable rate region is obtained from (3.1) while Han-Kobayashi scheme achievable rate region is given in [10].

In Fig. 3, the achievable rate region of MCMAC coincides with the one of the Gaussian interference channel. Based on the classification of two users symmetric interference channels in [18], we observe that using the interference channel approach to estimate the achievable rate region result in the 


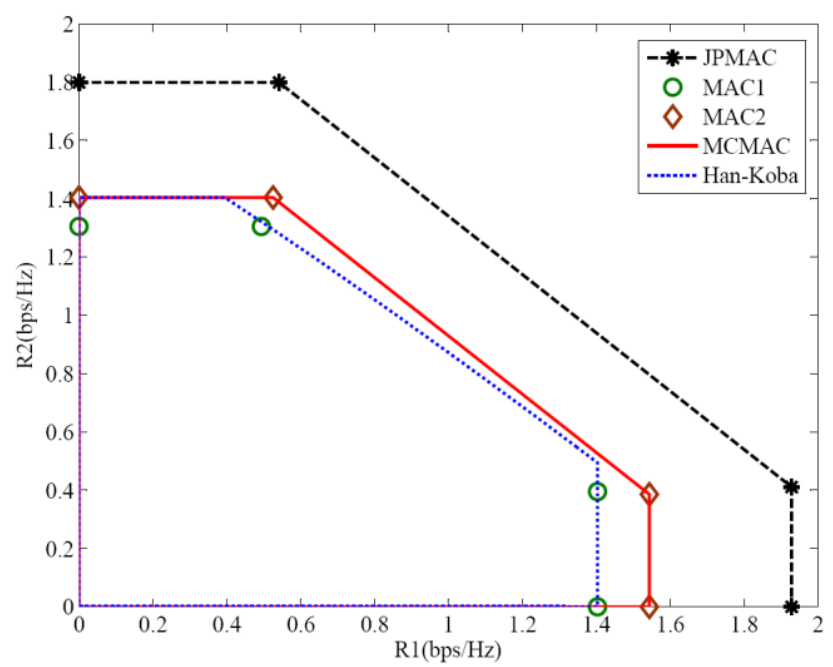

Fig. $5 P_{1}=P_{2}=5, h_{12}=0.85, h_{21}=1.25$

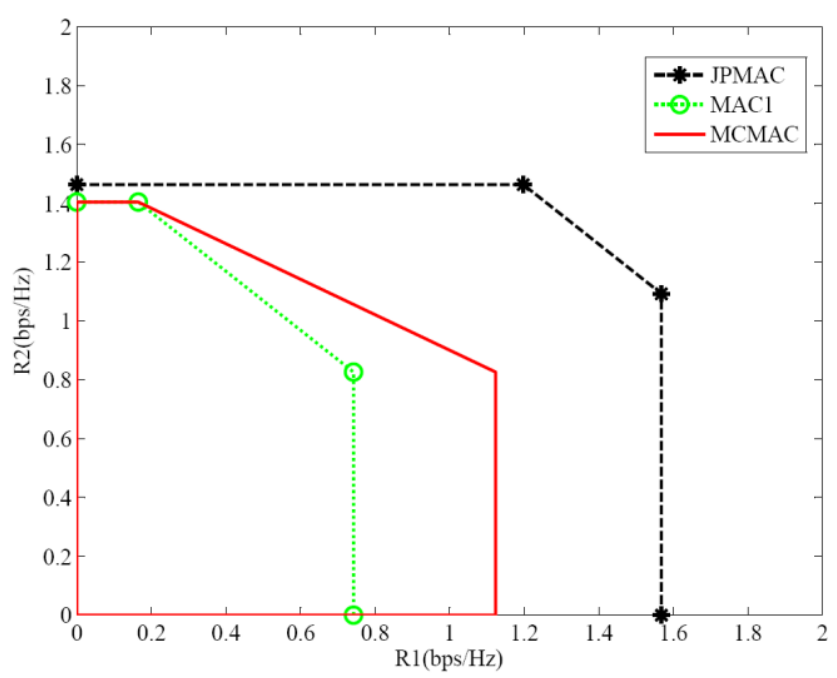

Fig. 6. Case 2: $P_{1}=P_{2}=6, h_{12}=0.1, h_{21}=0.3$

same MCMAC for $0 \leq h_{12}=h_{21} \leq 1$. In Fig. $4, h_{12}=h_{21}>1$ (the strong and very strong interference channel [8], [18]) and the achievable rate region of MCMAC extends beyond the achievable rate region of interference channels. Fig. 5 shows a non symmetric channel scenario where the channels coefficients of MAC1 $h_{11}$ and $h_{21}$ are better than these of MAC2 $h_{22}$ and $h_{12}$. The result shows that the achievable rate of MCMAC coincides with the one of MAC1 while the achievable rate region of the interference channel is within that of MCMAC.

Fig. 6 shows result for case 2 in which Receiver 1 can only decode the message of user 1 while receiver 2 can decode both messages. As long as $I\left(X_{1} ; Y_{1}\right)>I\left(X_{1} ; Y_{2}\right) \mid X_{2}$ the achievable rate region of MCMAC extends beyond that of MAC2, however, when $I\left(X_{1} ; Y_{1}\right) \leq I\left(X_{1} ; Y_{2}\right) \mid X_{2}$, the achievable rate region of MCMAC becomes the same one as MAC2.

\section{CONCLUSION}

In this paper, we have established the achievable rate region for MCMAC which is suboptimal in comparison with full cooperation but this technique requires less complexity than JPMAC. We established the dominating points in the achievable rate region using the polymatroid concept and indicated that MCMAC has an achievable rate region whose boundary is defined by time sharing between dominating points. Based on the Gaussian MCMAC analysis, our approach can result in significant sum-rate improvement over interference limited performance in the cellular system.

\section{REFERENCES}

[1] T. M. Cover, J. A. Thomas, "Elements of information theory," New York: Wiley, 2006

[2] R. Knopp and P.A. Humblet, "Information capacity and power control in single-cell multiuser communications," presented at the international conference of communications Seattle, WA, June 1995.

[3] W. Yu, W. Rhee, S. Boyd, J. M. Cioffi, "Iterative water-filling for Gaussian vector multiple-access channels," IEEE Trans. Inform. Theory, vol. 50, no. 1, pp. 145-152, Jan. 2004.

[4] D. N. C. Tse and S. V. Hanly, "Multiaccess fading channel-part I: polymatroid structure, optimal resource allocation and throughput capacities" IEEE Trans. Inform. Theory, vol. 44, no. 7, pp. 2796-2815, Nov. 1998.

[5] S. V. Hanly and D. N. C. Tse, "Multiaccess fading channel-part II: Delay limited capacities" IEEE Trans. Inform. Theory, vol. 44, no. 7, pp. 2816-2831, Nov. 1998.

[6] L.Li, N. Jindal, A. Goldsmith, "Outage capacity and optimal power allocation for fading multiple-access channels," IEEE Trans. Inform. Theory, vol. 51, no. 4, pp. 1326-1347, Apr. 2005.

[7] N. Jindal, S. Vishwanath, and A. Goldsmith, "On the duality of Gaussian multiple - access and broadcast channels," IEEE Trans. Inform. Theory, vol. 50, no. 5, pp. 768-783, May 2004.

[8] H. Sato, "The Capacity of the Gaussian interference channel under strong interference," IEEE Trans. Inform. Theory, vol. IT-27, no.6, pp. 786-788, Nov. 1981.

[9] A. B. Carleial, "Interference Channels," IEEE Trans. Inform. Theory, vol. IT-24, pp. 60-70, Jan 1978.

[10] T. S. Han and K. Kobayashi, "A new achievable rate region for the interference channels," IEEE Trans. Inform. Theory, vol. 27, no 1, pp. 49-60, Jan 1981.

[11] A. B. Carleial, "A case where interference does not reduce capacity," IEEE Trans. Inform. Theory, vol. IT-21, no.5, pp. 569-570, Sep. 1975.

[12] R. H. Etkin, D. N. C. Tse, and H. Wang, "Gaussian interference channel capacity to within one bit," IEEE Trans. Inform. Theory, vol. 54, no. 12, pp. 5534-5562, Dec. 2008.

[13] G. Bresler, A. Parekh, D. Tse , "The approximate capacity of many-toone and one-to-many Gaussian interference channels," in Proc. Allerton Conf. Communications, Control and Computing, Monticello, IL, Sep. 2007, pp. 791-801.

[14] A. D. Wyner, "Shannon-theoretic approach to a Gaussian cellular multiple-access channel," IEEE Trans. Inform. Theory, vol. 40, no. 6, pp. 1713-1727, Nov. 1994

[15] O. Somekh and S. Shamai, "Shannon-theoretic approach to a Gaussian cellular multiple-access channel with fading," IEEE Trans. Inform. Theory, vol. 46, no. 4, pp. 1401-1425, Jul. 2000.

[16] A. Sanderovich, O. Somekh, H. V. Poor and S. Shamai, "Uplink macro diversity of limited backhaul cellular network," IEEE Trans. Inform. Theory, vol. 55, no. 8, pp. 3457-3478, Jul. 2009.

[17] O. Simeone, O. Somekh, H. V. Poor and S. Shamai, "Local base station cooperation via finite-capacity links for the uplink of linear cellular networks," IEEE Trans. Inform. Theory, vol. 55, no. 1, pp. 190-204, Jan. 2009.

[18] M. H. M. Costa, "On the Gaussian Interference Channel”, IEEE Trans. Inform. Theory, vol. IT-31, no. 5, pp. 601-615, Sep. 1985 